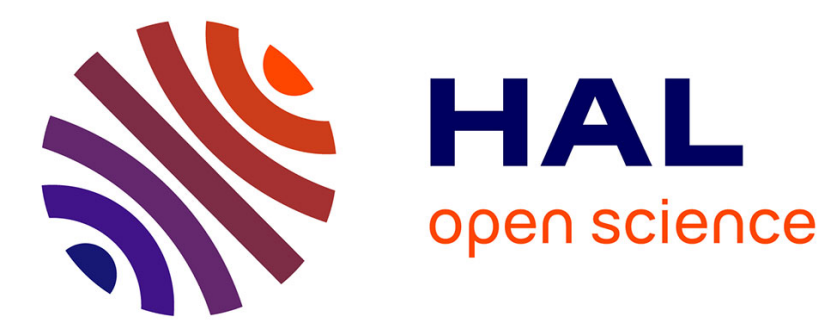

\title{
Mouvements de parois dans un nématique sous champ tournant
}

\author{
P.-G. de Gennes
}

\section{To cite this version:}

P.-G. de Gennes. Mouvements de parois dans un nématique sous champ tournant. Journal de Physique, 1971, 32 (10), pp.789-792. 10.1051/jphys:019710032010078900 . jpa-00207136

\section{HAL Id: jpa-00207136 https://hal.science/jpa-00207136}

Submitted on 1 Jan 1971

HAL is a multi-disciplinary open access archive for the deposit and dissemination of scientific research documents, whether they are published or not. The documents may come from teaching and research institutions in France or abroad, or from public or private research centers.
L'archive ouverte pluridisciplinaire HAL, est destinée au dépôt et à la diffusion de documents scientifiques de niveau recherche, publiés ou non, émanant des établissements d'enseignement et de recherche français ou étrangers, des laboratoires publics ou privés. 
Classification

Physics Abstracts :

14.82

\title{
MOUVEMENTS DE PAROIS DANS UN NÉMATIQUE SOUS CHAMP TOURNANT
}

\author{
P. G. de GENNES \\ Department of Physics, Harvard University (*), Cambridge, Mass.
}

(Reçu le 19 mars 1971)

\begin{abstract}
Résumé. - Soit une lame nématique dont une face $\left(F_{1}\right)$ est ancrée à une couche solide imposant une orientation fixe, l'autre $\left(\mathrm{F}_{2}\right)$ étant libre. La lame est soumise à un champ magnétique $H$ tournant. Si l'on parvient à éviter la nucléation de disclinations, on devrait observer un train de parois à $180^{\circ}$ migrant de $F_{1}$ vers $F_{2}$, d'où, dans des cas favorables, une coloration par rotation.

Abstract. - We consider a nematic slab, the molecules being anchored to one fixed direction on one side $\left(\mathrm{F}_{1}\right)$, but freely orienting in the limiting plane of the other side $\left(\mathrm{F}_{2}\right)$. A field $H_{1}$ rotating in the plane of the slab, is applied. Assuming that disclination loops do not nucleate, we discuss the apparition and migration of $180^{\circ}$ walls in the slab. In some favorable cases, the sample might become colored under the rotating field.
\end{abstract}

L'aspect de la lame envisagée est représenté sur la figure 1. Shtrikman et Fuller-Brown [1] ont noté, à partir des équations de Frank [2] qu'une telle lame, en présence d'un champ statique $H$, n'accepte pas de conformations (même métastables) pour lesquelles le nématique s'enroule d'un angle $\Delta \psi \geqslant \pi$ dans l'épaisseur. Nous montrons ici qu'avec un champ $\mathrm{H}$ tournant, on peut, par contre, avoir de forts enroulements.

Nous supposons que :

1) La face $F_{1}$ est suffisamment idéale pour que aucune boucle de disclination ne se forme à son contact (la nucléation de boucles en volume paraît plus difficile [3]).

2) La constante élastique de torsion [2] est plus petite que les autres, de façon à ce que les molécules restent constamment dans le plan de la lame.

Dans ce cas, la torsion imposée par le champ tournant ne peut relaxer que par nucléation de parois $\grave{a}$ $180^{\circ}$ sur la face $F_{1}$. En régime statique, les propriétés de telles parois ont été souvent discutées [4]. En régime dynamique l'angle $\psi$ entre les molécules et l'axe facile de $\mathrm{F}_{1}$ est régi par l'équation

$$
\theta \frac{\partial \psi}{\partial t}=\xi^{2} \frac{\partial^{2} \psi}{\partial z^{2}}-\frac{1}{2} \sin 2(\psi-\alpha(t))
$$

$\theta=\gamma_{1} / \chi_{\alpha} H^{2}$ est un temps caractéristique ; $\gamma_{1}$ est un coefficient de friction introduit par Leslie [5];

$\left(^{*}\right)$ Adresse permanente : Bât. 510, Faculté des Sciences, 91, Orsay, France.

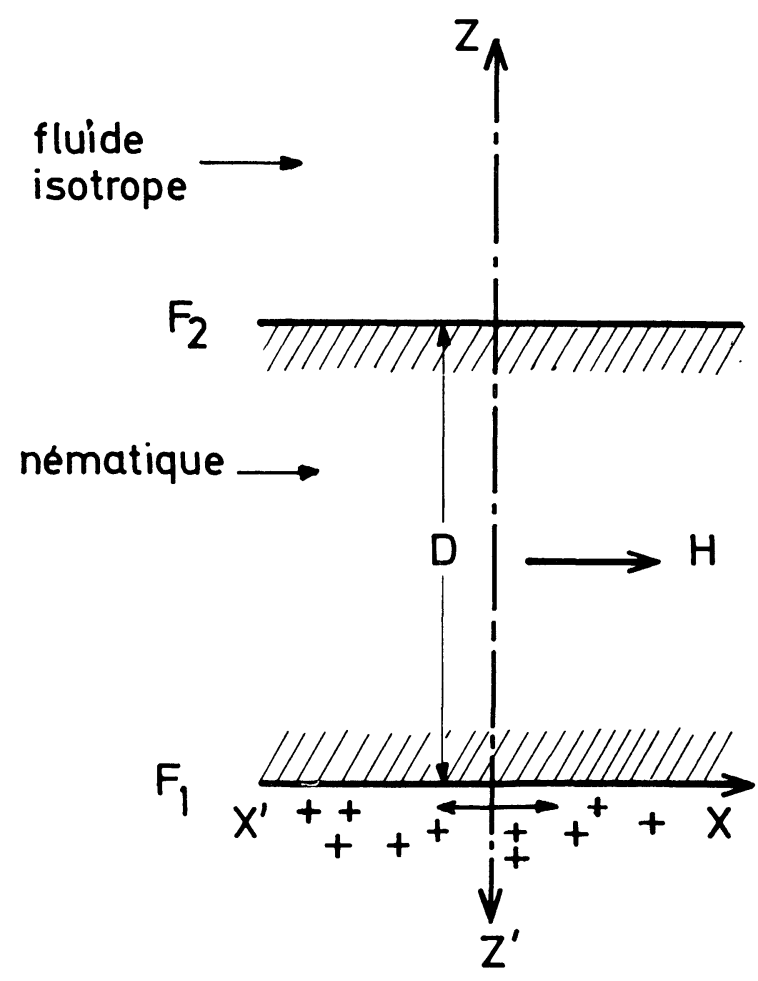

Fig. 1. - Le spécimen nématique est « ancré » sur un support solide en $\mathrm{F}_{1}$ (au contact de $\mathrm{F}_{1}$ les molécules nématiques doivent être parallèles à l'axe facile $x^{\prime} x$ ) et soumis au champ $H$. On peut soit faire tourner $H$ autour de $z z^{\prime}$, soit faire tourner la lame $\mathrm{F}_{1}$ (en maintenant $H$ fixe). On suppose que la condition aux limites sur la face libre $\mathrm{F}_{2}$ correspond à des molécules tangentes $\grave{a}$ l'interface. 
$\xi$ est la longueur de cohérence magnétique $[6] ; \alpha(t)$ est l'angle entre $H$ et l'axe facile de $\mathrm{F}_{1}$ : on peut varier $\alpha(t)$ soit en tournant $H$, soit en tournant $F_{1}$.

En l'absence d'ancrage aux frontières, le nématique effectue une rotation d'ensemble ( $\psi$ fonction de $t$ seul). Si $\alpha(t)=\omega t$, on a deux régimes selon la valeur de $\omega$ :

a) $\omega \theta<\frac{1}{2}$ : en régime permanent, le nématique tourne à même vitesse que $H$ :

$$
\left.\begin{array}{rl}
\psi & =\omega t+\psi_{0} \\
\sin 2 \psi_{0} & =-2 \omega \theta
\end{array}\right\}
$$

b) $\omega \theta>\frac{1}{2}$ : le nématique tourne moins vite que $H$ :

$$
\left.\begin{array}{rl}
\psi & =(\omega-\Omega) t+\text { termes oscillants } \\
\Omega \theta & =\left\{\frac{1}{\pi} \int_{0}^{\pi} \frac{\mathrm{d} \psi}{\omega \theta+\frac{1}{2} \sin 2 \psi}\right\}^{-1}
\end{array}\right\} .
$$

Mais, lorsque la face $\mathrm{F}_{1}$ est ancrée $(\psi(z=0, t)=0)$ les régimes sont plus complexes : c'est ce cas que nous voulons discuter ici.

1) Déplacement d'une paroi unique. - Partons du cas suivant :

$$
\alpha(t)=\left\{\begin{array}{lll}
\pi-\varepsilon & \text { pour } & t<0 \\
\pi & & t>0
\end{array} \text { avec } 0<\varepsilon \ll 1 .\right.
$$

a) La configuration initiale (pour $t<0$ ) choisie correspond à un enroulement de $\pi-\varepsilon$.

Elle est décrite par une fonction $\psi_{\varepsilon}\left(z-s_{0}\right)$ définie par :

$$
\tan \left(\frac{\psi_{\varepsilon}+\varepsilon}{2}\right)=\exp \left(\frac{z-s_{0}}{\xi}\right) .
$$

Il s'agit d'une paroi (d'épaisseur $\sim 2 \xi$ ) centrée sur $s_{0}$. La valeur de $s_{0}$ (déduite de la condition aux limites sur la face $\left.\mathrm{F}_{1}\right)$ est $s_{0}=\xi \ln (2 / \varepsilon)$.

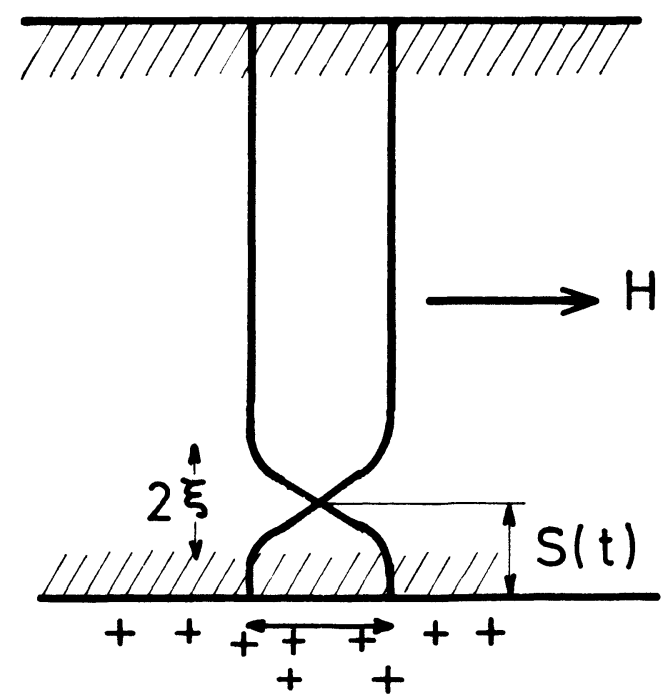

Fig. 2. - Arrangement moléculaire en présence d'une paroi à $180^{\circ}$ : au centre de la paroi $(z=s)$ les molécules sont normales au plan de la figure. b) Immédiatement après la rotation de $H$, toute la région entre la paroi et la face $F_{2}$ relaxe vers l'orientation $\pi$ en un temps microscopique de l'ordre de $\theta$.

c) La paroi est repoussée par la face $F_{1}$, et se déplace avec une certaine vitesse $v=\mathrm{d} s / \mathrm{d} t$. Nous calculons $v$ dans le cas $s_{0} \ll s \ll D$. Près de $F_{1}, \psi$ est petit, l'équation (1) est pratiquement linéaire et on peut satisfaire à la condition aux limites par une méthode d'images.

$$
\psi \cong \psi_{0}(z-s)-\psi_{0}(-z-s) .
$$

L'énergie correspondante est :

$$
\begin{aligned}
U & =\frac{1}{2} \chi_{a} H^{2} \int_{0}^{\infty} \mathrm{d} z\left[\xi^{2} \frac{\partial \psi^{2}}{\partial z}+\sin ^{2} \psi\right] \\
& =U_{0}+4 \chi_{a} H^{2} \mathrm{e}^{-2 s / \xi} \xi
\end{aligned}
$$

d'où la force de répulsion

$$
-\frac{\mathrm{d} U}{\mathrm{~d} s}=8 \chi_{a} H^{2} \mathrm{e}^{-2 s / \xi} .
$$

La puissance dissipée par friction vaut

$$
\gamma_{1} \int \mathrm{d} z\left(\frac{\partial \psi^{2}}{\partial t}\right) \simeq \gamma_{1} v^{2} \int\left(\frac{\partial \psi}{\partial z}\right)^{2} \mathrm{~d} z \simeq \frac{2 \gamma_{1} v^{2}}{\xi} .
$$

En égalant la force de friction $2 \gamma_{1} v / \xi$ à la répulsion (4) on obtient

$$
v=\frac{4 \xi}{\theta} \mathrm{e}^{-2 s / \xi}
$$

donc $s(t)$ doit croître logarithmiquement avec $t$. Typiquement $H=10^{4}$ gauss, $\chi_{a}=10^{-7}, \gamma_{1}=0,1$ poise, $K_{2}=10^{-6}$ dyne, d'où $\xi \simeq 3$ microns et $\theta \simeq 10^{-2} \mathrm{~s}$.

2) Train de parois. - Avec un champ tournant lent $(\alpha=\omega t$ et $2 \omega \theta<1)$, il est plausible d'admettre qu'il apparaît sur la face $F_{1}$ un nombre de parois (par s.) $J=\omega / \pi$. Ces parois se déplacent vers $F_{2}$ avec une vitesse $v(z)$ (lentement croissante avec $z$ ). L'intervalle entre parois $L(z)$ est lié à $v(z)$ par la loi de conservation

$$
J=\frac{\omega}{\pi}=\frac{v(z)}{L(z)} .
$$

Localement (à l'échelle de quelques parois) la solution de (1) est approximativement de la forme

$$
\psi(z t)=\omega t+\varphi(z-v t) .
$$

La condition (6) exprime que, à $z$ fixé, $\psi$ oscille autour d'une moyenne $\bar{\psi}(z)$ indépendante de $t[7]$.

L'énergie par paroi vaut :

$$
U=\frac{1}{2} K_{2} \int_{z}^{z+L}\left[\left(\frac{\partial \varphi}{\mathrm{d} z}\right)^{2}+\xi^{-2} \sin ^{2} \varphi\right] \mathrm{d} z
$$

$\left(K_{2}=\right.$ constante élastique de torsion [2]). Dans l'approximation statique ( $\omega$ et $v$ étant provisoirement négligés) l'équation pour $\varphi$ a l'intégrale première.

$$
\xi^{2}\left(\frac{\mathrm{d} \varphi}{\mathrm{d} z}\right)^{2}+\sin ^{2} \varphi=2 E .
$$




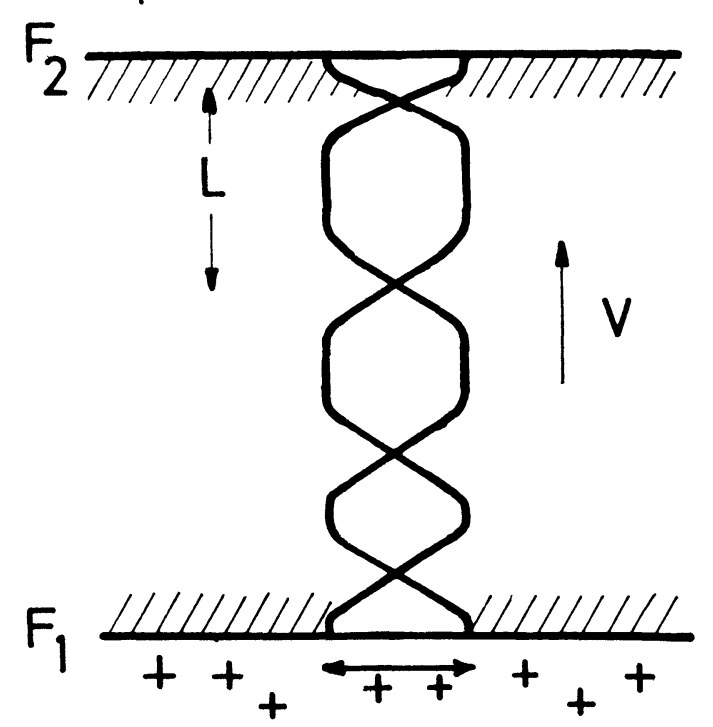

Fig. 3. - Train de parois se déplaçant de $F_{1}$ vers $F_{2}$, «photographié » au moment où le champ tournant $H$ est parallèle à l'axe facile de la plaque $F_{1}$.

La distance entre parois $L$ est alors reliée à la constante $E$ par la condition

$$
\begin{aligned}
L & =\int_{0}^{\pi} \frac{\mathrm{d} z}{\mathrm{~d} \psi} \mathrm{d} \psi \\
& =\xi \int_{0}^{\pi} \frac{\mathrm{d} \psi}{\sqrt{2 E+\sin ^{2} \psi}} .
\end{aligned}
$$

L'éq. (10) peut être exprimée en termes d'intégrales elliptiques. Elle se simplifie dans ceux cas limites :

$$
\begin{array}{ll}
L>\xi & E \rightarrow 8 \exp \left(\frac{-L}{\xi}\right) \\
L<\xi & E \rightarrow \frac{\pi^{2}}{2}\left(\frac{\xi}{L}\right)^{2} .
\end{array}
$$

On tire de (8) et (9) une expression pour la pression $p$ du « cristal de parois » $[8]$ :

$$
p=-\frac{\partial U}{\partial L}=\chi_{a} H^{2} E .
$$

Si $L$ varie avec $z$, il en est de même pour $E$ et $p$, donc il apparaît une force par unité de volume $-\partial p / \partial z$, due aux interactions élastiques entre parois. Chaque paroi subit une force $\left(\right.$ par $\left.\mathrm{cm}^{2}\right)-L \partial p / \partial z$. Le travail de cette force doit être retrouvé sous forme de chaleur dissipée

$$
-L \frac{\partial p}{\partial z} v=\gamma_{1} v^{2} \int_{z}^{z+L}\left(\frac{\mathrm{d} \psi}{\mathrm{d} z}\right)^{2} \mathrm{~d} z=\frac{\gamma_{1} v^{2}}{\xi} I(E)
$$

avec

$$
I(E)=\int_{0}^{\pi} \mathrm{d} \psi\left(2 E+\sin ^{2} \psi\right)^{1 / 2} .
$$

En utilisant (6) et (12), on peut récrire ces résultats sous la forme

$$
-\frac{\partial p}{\partial z}=\frac{\gamma_{1} \omega}{\pi \xi} I(E)=\chi_{a} H^{2} \frac{\partial E}{\partial z} .
$$

Pour intégrer l'éq. (15), il faut disposer d'une condition aux limites "macroscopiques" pour le cristal de parois sur la face $\mathrm{F}_{2}(z=D)$. Si $F_{2}$ est un interface nématique/milieu isotrope (imposant la condition microscopique $\partial \psi / \partial z(z=D)=0)$ aucune barrière ne s'oppose à l'évacuation des parois, et il est raisonnable de prendre

$$
p(z=D)=0 .
$$

Donc $E(z=D)=0$ d'après (12) et l'intégration de (15) donne

$$
\frac{D-z}{\xi}=\frac{\pi}{\omega \theta} \int_{0}^{E(z)} \frac{\mathrm{d} E^{\prime}}{I\left(E^{\prime}\right)} .
$$

Cette équation définit $E(z)$, et donc $L(z)$ (d'après l'éq. 10), c'est-à-dire la forme spatiale du train de parois. Pour l'expliciter, nous utiliserons seulement les cas limites $(11 a, b)$.

a) Si $\omega \pi<\xi / D$ on peut vérifier en fin de calcul que $L$ est partout plus grand que $\xi$ (parois espacées). Dans ce régime $I(E) \sim 2$ et $\partial p / \partial z$ est indépendant de $z$. A partir de (14) et (11a) on obtient :

$$
\frac{L(z)}{\xi}=\log \left(\frac{\xi}{D-z} \frac{4 \pi}{\omega \theta}\right) .
$$

b) $\operatorname{Si} \omega \theta>\xi / D, L$ est presque partout inférieur à $\xi$, et donné par :

$$
\frac{L(z)}{\xi}=\frac{\pi \xi}{(D-z) \omega \theta} .
$$

Les équations (18) montrent que l'on peut avoir de forts enroulements (en particulier pour $D$ grand) en régime dynamique. Par exemple, dans le cas $(b)$, la période la plus courte (réalisée au voisinage de $F_{1}$ ) vaut :

$$
L(0)=\frac{\pi \xi^{2}}{D \omega \theta}=\frac{\pi K_{2}}{\gamma_{1} \omega D} \quad\left(\omega \theta>\frac{\xi}{D}\right) .
$$

Si $\omega=10$ (100 tours $/ \mathrm{mn}), K_{2}=10^{-6}$ dynes, $\gamma_{1}=0,1$ poise et $D=1 \mathrm{~mm}$, ceci correspond à $2 L(0) \sim 0,6$ micron : dans un tel cas (si on n'est pas gêné par la nucléation de disclinations) le spécimen doit donner, près de la face $F_{1}$, des réflexions de Bragg dans le domaine visible, donc se colorer par rotation [9].

L'équation (19) ne fait pas intervenir la valeur de $H$. Mais notre description du train de parois (éq. 6 et 7 ) n'est applicable que si la condition d' " accrochage» $\omega \theta<1 / 2$ est satisfaisante, soit

$$
H>\sqrt{\frac{2 \gamma_{1} \omega}{\chi_{a}}} .
$$


Avec lés valeurs citées plus haut, ce seuil serait de 5000 gauss. Donc l'expérience paraît possible; on pourrait détecter le passage des parois par plusieurs méthodes, notamment :

— pour $D$ grand : étude de la réflexion sélective sur la face $\mathrm{F}_{1}$;
- pour $D$ plus petit ( 30 microns) $\vdots$ étude de la rotation optique subie par un faisceau traversant la lame.

Remerciements. - Nous avons bénéficié de discussions très stimulantes avec R. B. Meyer, S. Shtrikman, M. Papoular et L. Levelut.

\section{Bibliographie}

[1] Shtrikman (S.), Communication privée.

[2] Frank (F. C.), Faraday Soc. Disc., 1958, 25, 19.

[3] La nucléation de boucles de disclination dans un nématique sous torsion est discutée dans la référence suivante : FRIEDEL (J.), DE GENNES (P. G.), C. R. Acad. Sci. Paris, 1969, 268, 257.

[4] Voir par exemple Helfrich (W.), Phys. Rev. Letters, $1969,22,1342$. Il est probable que l'expérience de Williams, discutée dans cette référence, ne fait pas intervenir de parois, mais l'analyse théorique reste valable par $\mathrm{s}$.

[5] Leslie (F. M.), Arch. Rat. Mech. Anal., 1968, 28, 265.

[6] Voir par exemple DE GenNes (P. G.), Molecular Crystals, 1969,7 , p. 325.

[7] L'équation pour $(\varphi)$ a la forme de l'équation d'un pendule soumis à un couple extérieur $(\omega)$ et à un frottement visqueux $(v), z$ jouant le rôle du temps.
Cette remarque conduit à une autre dérivation de l'éq. (15), et aussi de l'équation (5).

[8] II peut être utile de souligner que $p(z)$, qui mesure les répulsions entre parois, n'a rien à voir avec la pression hydrostatique au point $(z)$.

[9] Une géométrie légèrement différente et peut-être techniquement plus commode nous a été suggérée par le Professeur R. B. Meyer : elle consiste à utiliser deux plaques polies $F_{1}$ et $F_{1}^{\prime}$ (de même axe facile), le nématique occupant tout l'intervalle entre $F_{1}$ et $F_{1}^{\prime}$. Alors, en tournant les plaques dans un champ $H$ fixe, on pourrait engendre des parois (de signe opposé sur les deux faces) qui migrent vers le centre et s'y annihilent par paires. 